\title{
Knowledge, attitudes and practice patterns of patients with diabetes mellitus and diabetic retinopathy in an urban eye clinic in Singapore
}

\author{
Elin Lee, Srinivasan Sanjay, Tiakumzuk Sangtam \\ Department of Ophthalmology and Visual Sciences (OVS), Khoo Teck Puat \\ Hospital, Alexandra Health, 90 Yishun Central, Singapore 768828.
}

\begin{abstract}
Background: Diabetes mellitus (DM) is a global health concern, the prevalence of which is predicted to increase in the coming years. Consequently, the prevalence of diabetic retinopathy $(D R)$ is expected to rise. Therefore, identification of prevailing knowledge, attitudes and practice patterns (KAPP) of patients to address knowledge gaps and provide preventive education about $D M$ and $D R$ could reduce potentially sight-threatening complications. Our objectives were to assess KAPP of patients with DM and DR, factors influencing KAPP and impact of education on their KAPP.

Methods: A questionnaire based survey among diabetic patients in an eye clinic in Singapore. A response rate of $92.6 \%$ was achieved. Participants were administered a set of questions about KAPP on DM and DR before and after educating them about $D R$.

Results: Knowledge of DM and DR was noted in $57.0 \%$ and $29.0 \%$ of participants respectively. Good attitude and practice patterns were found in nearly $60 \%$ and above of the patients. Participants with more knowledge base had better attitude and practice patterns. Higher educational level, Indian ethnicity and longer duration of DM had a positive influence on KAPP. There was a statistically significant $(p<0.05)$ impact of education on KAPP among patients with DM and DR.

Conclusions: The knowledge, attitude and practice patterns among patients were variable. A low proportion of knowledge of $D R$ was noted which increased to almost three times after education. There is a need to actively implement educational strategies among patients with $D M$ and $D R$.
\end{abstract}

Key words: Knowledge, attitudes and practice patterns (KAPP), diabetes mellitus, diabetic retinopathy, Singapore

\section{Introduction}

Diabetes mellitus (DM) is a major health issue. Yet, there appears to be poor knowledge about DM and diabetic retinopathy (DR) among diabetic patients. ${ }^{1,2}$

Several studies on knowledge, attitudes and practice patterns (KAPP) of patients with DM and DR have been published in many countries ${ }^{3,4}$. A local study in multiethnic Singapore explored the awareness of DM and DR, while another report ${ }^{1}$ assessed the knowledge and practice of DM. However, a sole study combining

Correspondence: Dr. Srinivasan Sanjay, Ophthalmology and Visual Sciences, Khoo Teck Puat Hospital, Alexandra Health, 90 Yishun Central, Singapore 768828.

E-mail: sanjay_s@alexandrahealth.sg 
KAPP of patients with DM and DR among all ethnic groups is lacking. In our pilot study, we conducted an assessment of KAPP in all ethnic groups (Chinese, Indian and Malays), factors influencing KAPP and the impact of education on KAPP.

\section{Methods}

\section{Design}

Every third diabetic patient seeking eye treatment (diabetic-related or -unrelated eye problems) at an urban eye clinic in Singapore was enrolled to complete the questionnaire. One hundred valid responses out of one hundred and eight respondents were obtained, giving a response rate of $92.6 \%$.

\section{Participants}

Any diabetic patient with good mental capacity to answer the questionnaire was included. Diabetic patient, for the purpose of this study, was defined as either using diabetic medication or had a previous physician diagnosis of diabetes.

\section{Questionnaire}

This study was conducted using an interview-based questionnaire. The text was standardized in English and Chinese. A stand-by translator was assigned for Malay and Tamil version.

The questionnaire was divided into five sections:

- Section A: Demographic information

- Section B: Socio-economic status

- Section C: Knowledge

- Section D: Attitude

- Section E: Practice Patterns

After the completion of Sections A to E, participants were educated during approximately twenty minutes about DR, using a booklet entitled Management of Diabetic Retinopathy written and published by the Singapore Health Promotion Board (HPB). ${ }^{6}$ Then the questions from Sections $C$ to $E$ were repeated to assess the impact of education on KAPP.

\section{Retinal photograph and diabetic retinopathy assessment}

Information regarding the status of DR of patients was recorded by retinal photography. The severity of DR was categorized as defined by the Early Treatment Diabetic Retinopathy Study. ${ }^{7}$ Additionally, participants were assessed if they correctly knew the severity of DR they had, as part of knowledge component. Correct responses were categorized when the recorded severities during retinal assessment matched patients' responses.

\section{Definitions}

Knowledge of DM: This was defined if participants:

- Have heard of DM and

- Agree that DM is familial. 
Knowledge of DR: This was defined if participants:

- Have heard of DR and

- Agree vision is affected in DR.

Attitude: Good attitude was defined if participants agreed that:

- Diabetic patients should go for eye examinations and

- If so, the frequency would usually be annually or as per the advice of the physician.

Practice Patterns: Good practice pattern was defined if participants agreed that:

- Diabetic patients should visit an eye doctor despite good blood sugar control and

- An eye doctor should be their choice of healthcare professional should they be diagnosed with DR.

Since eye health is involved, an eye doctor was designated as the gold standard of care.

\section{Statistical analysis}

Data were analyzed using the Statistical Package for the Social Sciences (SPSS) software version 17.0.

Univariate analysis was performed to examine the distribution of the demographic and socio-economic status of the study population as well as determinants of KAPPa such as gender, age, ethnicity, educational level, housing type and duration of DM. This was followed by multiple logistic regression analysis. The association of knowledge of DR with attitude and practice patterns was evaluated between the groups using bivariate analysis (Chi-square test). The correlation between KAPPa and the factors influencing them were evaluated using Spearman rank correlation test. Paired t-test (McNemar's test) was used to assess the impact of education on KAPPa. A two-tailed ' $p$ ' value of $<0.05$ was considered statistically significant for all analyses. The $95 \%$ confidence intervals $(\mathrm{Cl})$ of the estimates were calculated by assuming normal approximation of binomial distribution for prevalence of one percent or more.

\section{Ethical considerations and statement of ethics}

As this was a Quality Improvement Study, no approval from the Institutional Review Board (IRB) was needed.

\section{Results}

\section{Demographic and socio-economic data}

All one hundred respondents were above 21 years (median age $=62$ years; range $=$ 28-88 years) with a slight preponderance of males comprising $56.0 \%$ of the sample population.

The majority of participants were married $(81 \%)$, having primary or lower than primary education level (67\%), not working (53\%), earning an average household income of Singapore Dollar (SG\$) 2,000 and below (71\%) and living in Housing Development Board (HDB) flats (94\%). 


\section{Findings on KAPP}

Pre-existing knowledge of DM and DR were noted in 57 (57.0 \%) and 29 (29.0\%) participants respectively. Thirty-five percent of participants had combined knowledge of DM and DR. Interestingly, $21 \%$ of participants knew about DR despite not having any knowledge of DM.

The attitude that diabetic patients should go for eye examination was noted in 78 (78.0\%) and that it should be done usually on an annual basis was noted in 46 out of these 78 participants (59.0\%).

The practice patterns of patients that they should or will visit an ophthalmologist even if they had good blood glucose control was noted in 66 participants $(66.0 \%)$. The practice of choosing an eye doctor as their healthcare professional if they were diagnosed with DR was noted in 59 participants (59.0\%).

\section{Factors influencing KAPP}

\section{a. Factors influencing knowledge of DM and DR (Table 1)}

Knowledge of DM was significantly higher among Indians compared to Chinese [Odds Ratio (OR): 6.73; 95\% confidence interval (Cl) 1.59-28.39; overall p-value: 0.03]. Knowledge of DR was shown to be higher in those who had more than ten years duration of DM compared to zero to five years duration of DM (OR: $1.27 ; 95 \% \mathrm{Cl}$ : 0.38-4.24; overall $p$-value: 0.05 ).

Table 1. Associations of knowledge of DM and DR with participant characteristics, KAPP of DM and DR in a tertiary eye clinic in Singapore.

\begin{tabular}{|c|c|c|c|c|c|c|c|}
\hline \multirow[t]{2}{*}{ Variable } & \multirow[t]{2}{*}{ Total } & \multicolumn{3}{|c|}{ Knowledge of DM } & \multicolumn{3}{|c|}{ Knowledge of DR } \\
\hline & & n (\%) & p-value & $\begin{array}{l}\text { Adjusted } \\
\mathrm{OR}^{2} \\
\left(95 \% \mathrm{Cl}^{3}\right)\end{array}$ & n (\%) & p-value & $\begin{array}{l}\text { Adjusted } \\
\text { OR } \\
(95 \% \mathrm{Cl})\end{array}$ \\
\hline \multicolumn{8}{|l|}{ Gender } \\
\hline Male & 56 & $29(51.8)$ & - & 1.00 & $18(32.1)$ & - & 1.00 \\
\hline Female & 44 & $28(63.6)$ & 0.10 & $\begin{array}{l}2.36 \\
(0.84-6.60)\end{array}$ & $11(25.0)$ & 0.21 & $\begin{array}{l}0.40 \\
(0.10-1.65)\end{array}$ \\
\hline \multicolumn{8}{|c|}{ Age group (yrs) } \\
\hline $21-59$ yrs & 41 & $26(63.4)$ & - & 1.00 & $12(29.3)$ & - & 1.00 \\
\hline $60 \mathrm{yrs}$ & 59 & $31(52.5)$ & 0.30 & $\begin{array}{l}0.61 \\
(0.24-1.57)\end{array}$ & $17(28.8)$ & 0.60 & $\begin{array}{l}0.74 \\
(0.24-2.30)\end{array}$ \\
\hline Ethnic group & & & 0.03 & & & 0.91 & \\
\hline Chinese & 71 & $35(49.3)$ & - & 1.00 & $21(29.6)$ & - & 1.00 \\
\hline Malay & 11 & $7(63.6)$ & 0.40 & $\begin{array}{l}1.94 \\
(0.41-9.20)\end{array}$ & $3(27.3)$ & 0.71 & $\begin{array}{l}1.44 \\
(0.22-9.67)\end{array}$ \\
\hline
\end{tabular}




\begin{tabular}{|c|c|c|c|c|c|c|c|}
\hline Variable & Total & \multicolumn{3}{|c|}{ Knowledge of DM } & \multicolumn{3}{|c|}{ Knowledge of DR } \\
\hline & & n (\%) & p-value & $\begin{array}{l}\text { Adjusted } \\
\text { OR }^{2} \\
\left(95 \% \mathrm{Cl}^{3}\right)\end{array}$ & n (\%) & p-value & $\begin{array}{l}\text { Adjusted } \\
\text { OR } \\
(95 \% \mathrm{Cl})\end{array}$ \\
\hline Indian & 18 & $15(83.3)$ & 0.01 & $\begin{array}{l}6.73 \\
(1.59-28.39)\end{array}$ & $5(27.8)$ & 0.78 & $\begin{array}{l}1.23 \\
(0.30-5.01)\end{array}$ \\
\hline Educational level $^{4}$ & & & 0.67 & & & 0.004 & \\
\hline $\begin{array}{l}\text { No formal } \\
\text { qualifications/ } \\
\text { Lower primary }\end{array}$ & 30 & $16(53.3)$ & - & 1.00 & $10(33.3)$ & - & 1.00 \\
\hline Primary (PSLE) & 37 & $22(59.5)$ & 0.96 & $\begin{array}{l}1.03 \\
(0.31-3.43)\end{array}$ & $4(10.8)$ & 0.003 & $\begin{array}{l}0.06 \\
(0.008-0.37)\end{array}$ \\
\hline $\begin{array}{l}\text { Secondary ('O'/' }{ }^{\prime} \mathrm{N}^{\prime} \\
\text { level) }\end{array}$ & 18 & $12(66.7)$ & 0.52 & $\begin{array}{l}1.59 \\
(0.38-6.65)\end{array}$ & $8(44.4)$ & 0.92 & $\begin{array}{l}0.91 \\
(0.17-4.82)\end{array}$ \\
\hline Post-Secondary & 15 & $7(46.7)$ & 0.49 & $\begin{array}{l}0.57 \\
(0.12-2.79)\end{array}$ & $7(46.7)$ & 0.76 & $\begin{array}{l}0.75 \\
(0.12-4.67)\end{array}$ \\
\hline Housing type & & & 0.83 & & & 0.03 & \\
\hline $\begin{array}{l}\mathrm{HDB}^{1} 1 \text { \& } 2 \text { room(s) } \\
\text { flat }\end{array}$ & 7 & $4(57.1)$ & - & 1.00 & $3(42.9)$ & - & 1.00 \\
\hline HDB 3 room flat & 19 & $11(57.9)$ & 0.10 & $\begin{array}{l}1.00 \\
(0.14-7.39)\end{array}$ & $3(15.8)$ & 0.07 & $\begin{array}{l}0.11 \\
(0.01-1.25)\end{array}$ \\
\hline HDB 4 room flat & 36 & $19(52.8)$ & 0.62 & $\begin{array}{l}0.62 \\
(0.09-4.18)\end{array}$ & 7 (19.4) & 0.12 & $\begin{array}{l}0.16 \\
(0.02-1.55)\end{array}$ \\
\hline HDB 5 room flat & 32 & $19(59.4)$ & 0.99 & $\begin{array}{l}0.99 \\
(0.15-6.71)\end{array}$ & $13(40.6)$ & 0.10 & $\begin{array}{l}0.10 \\
(0.11-8.93)\end{array}$ \\
\hline $\begin{array}{l}\text { Condominium and } \\
\text { Landed Property }\end{array}$ & 6 & $4(66.7)$ & 0.68 & $\begin{array}{l}1.74 \\
(0.13-23.38)\end{array}$ & $3(50.0)$ & 0.95 & $\begin{array}{l}0.91 \\
(0.06-13.74)\end{array}$ \\
\hline Duration of DM & & & 0.19 & & & 0.05 & \\
\hline $0-5$ years & 34 & $17(50.0)$ & & 1.00 & $11(32.4)$ & - & 1.00 \\
\hline $6-10$ years & 21 & $14(66.7)$ & 0.08 & $\begin{array}{l}3.12 \\
(0.86-11.29)\end{array}$ & $4(19.1)$ & 0.04 & $\begin{array}{l}0.18 \\
(0.04-0.90)\end{array}$ \\
\hline 10 years & 45 & $\begin{array}{l}26 \\
(57.8 .)\end{array}$ & 0.23 & $\begin{array}{l}1.88 \\
(0.67-5.27)\end{array}$ & $14(31.1)$ & 0.70 & $\begin{array}{l}1.27 \\
(0.38-4.24)\end{array}$ \\
\hline
\end{tabular}

${ }^{1} \mathrm{HDB}$ : Housing Development Board

2OR: odds ratio

${ }^{3} 95 \% \mathrm{Cl}: 95 \%$ confidence interval

${ }^{4}$ Lower primary: $<6$ years of formal education.

Primary: 6 years of formal education with attainment of Primary School Leaving Examination (PSLE) qualification. 
Secondary: 10-11 years of formal education with attainment of Ordinary or Normal level qualification.

Post-secondary: Attainment of advanced level and/or higher qualification.

Adjusted for gender, age, ethnic group, education, type of housing and duration of DM.

\section{b. Factors influencing attitude (Table 2)}

The attitude that diabetic patients should go for annual eye examinations was found to be significantly higher in those with secondary education (OR: $6.24 ; 95 \%$ Cl: 1.03-37.78; overall p-value: 0.03 ) and post-secondary education (OR: $8.94 ; 95 \% \mathrm{Cl}$ : 0.96-82.96; overall p-value: 0.03) compared to those with no formal or lower primary education. Furthermore, those with more than ten years duration of DM had better attitude for annual eye examination compared to those with zero to five years duration of DM (OR: 1.33; 95\% Cl: 0.35-5.04; overall p-value: 0.02).

Table 2. Associations of attitude with participant characteristics, KAP of DM and DR in a tertiary eye clinic in Singapore.

\begin{tabular}{|c|c|c|c|c|c|c|c|c|}
\hline \multicolumn{2}{|l|}{ Variable } & \multicolumn{7}{|c|}{ Attitude } \\
\hline & & \multicolumn{3}{|c|}{$\begin{array}{l}\text { Participants who agreed } \\
\text { diabetic patients should go } \\
\text { for eye examinations }\end{array}$} & & \multicolumn{3}{|c|}{$\begin{array}{l}\text { Participants who agreed } \\
\text { diabetic patients should } \\
\text { go for annual eye } \\
\text { examinations }\end{array}$} \\
\hline & Total & n (\%) & p-value & $\begin{array}{l}\text { Adjusted } \\
\mathrm{OR}^{2} \\
\left(95 \% \mathrm{Cl}^{3}\right)\end{array}$ & Total & n (\%) & p-value & $\begin{array}{l}\text { Adjusted } \\
\text { OR }(95 \% \\
\text { CI) }\end{array}$ \\
\hline \multicolumn{9}{|l|}{ Gender } \\
\hline Male & 56 & $\begin{array}{l}45 \\
(80.4)\end{array}$ & - & 1.00 & 45 & $\begin{array}{l}29 \\
(64.4)\end{array}$ & - & 1.00 \\
\hline Female & 44 & $\begin{array}{l}33 \\
(75.0)\end{array}$ & 0.85 & $\begin{array}{l}0.88 \\
(0.25-3.15)\end{array}$ & 33 & $\begin{array}{l}17 \\
(51.5)\end{array}$ & 0.29 & $\begin{array}{l}0.46 \\
(0.11-1.95)\end{array}$ \\
\hline \multicolumn{9}{|c|}{ Age group (yrs) } \\
\hline $21-59 y r s$ & 41 & $\begin{array}{l}36 \\
(87.8)\end{array}$ & - & 1.00 & 36 & $\begin{array}{l}19 \\
(52.8)\end{array}$ & - & 1.00 \\
\hline$>60$ yrs & 59 & $\begin{array}{l}42 \\
(71.2)\end{array}$ & 0.06 & $\begin{array}{l}0.28 \\
(0.08-1.04)\end{array}$ & 42 & $\begin{array}{l}27 \\
(64.3)\end{array}$ & 0.227 & $\begin{array}{l}2.07 \\
(0.64-6.69)\end{array}$ \\
\hline Ethnic group & & & 0.23 & & & & 0.53 & \\
\hline Chinese & 71 & $\begin{array}{l}53 \\
(74.7)\end{array}$ & - & 1.00 & 53 & $\begin{array}{l}32 \\
(60.4)\end{array}$ & - & 1.00 \\
\hline Malay & 11 & $8(72.7)$ & 0.80 & $\begin{array}{l}0.80 \\
(0.14-4.48)\end{array}$ & 8 & $3(37.5)$ & 0.27 & $\begin{array}{l}0.28 \\
(0.03-2.63)\end{array}$ \\
\hline
\end{tabular}




\begin{tabular}{|c|c|c|c|c|c|c|c|c|}
\hline \multicolumn{2}{|l|}{ Variable } & \multicolumn{7}{|c|}{ Attitude } \\
\hline & & \multicolumn{3}{|c|}{$\begin{array}{l}\text { Participants who agreed } \\
\text { diabetic patients should go } \\
\text { for eye examinations }\end{array}$} & & \multicolumn{3}{|c|}{$\begin{array}{l}\text { Participants who agreed } \\
\text { diabetic patients should } \\
\text { go for annual eye } \\
\text { examinations }\end{array}$} \\
\hline & Total & n (\%) & p-value & $\begin{array}{l}\text { Adjusted } \\
\text { OR }^{2} \\
\left(95 \% \mathrm{Cl}^{3}\right)\end{array}$ & Total & n (\%) & p-value & $\begin{array}{l}\text { Adjusted } \\
\text { OR }(95 \% \\
\text { CI) }\end{array}$ \\
\hline Indian & 18 & $\begin{array}{l}17 \\
(94.4)\end{array}$ & 0.09 & $\begin{array}{l}8.18(0.71- \\
93.94)\end{array}$ & 17 & $\begin{array}{l}11 \\
(64.7)\end{array}$ & 0.97 & $\begin{array}{l}0.97 \\
(0.23-4.07)\end{array}$ \\
\hline Educational level $^{4}$ & & & 0.34 & & & & 0.03 & \\
\hline $\begin{array}{l}\text { No formal } \\
\text { qualifications/ } \\
\text { Lower primary }\end{array}$ & 30 & $\begin{array}{l}20 \\
(66.7)\end{array}$ & - & 1.00 & 20 & $\begin{array}{l}10 \\
(50.0)\end{array}$ & - & 1.00 \\
\hline Primary (PSLE) & 37 & $\begin{array}{l}29 \\
(78.4)\end{array}$ & 0.92 & $\begin{array}{l}0.93 \\
(0.24-3.70)\end{array}$ & 29 & $\begin{array}{l}15 \\
(51.7)\end{array}$ & 0.64 & $\begin{array}{l}0.67 \\
(0.13-3.50)\end{array}$ \\
\hline $\begin{array}{l}\text { Secondary ('O'/' } \mathrm{N}^{\prime} \\
\text { level) }\end{array}$ & 18 & $\begin{array}{l}15 \\
(83.3)\end{array}$ & 0.65 & $\begin{array}{l}1.50 \\
(0.26-8.59)\end{array}$ & 15 & $\begin{array}{l}11 \\
(73.3)\end{array}$ & 0.05 & $\begin{array}{l}6.24(1.03- \\
37.78)\end{array}$ \\
\hline Post-Secondary & 15 & $\begin{array}{l}14 \\
(93.3)\end{array}$ & 0.09 & $\begin{array}{l}9.64(0.70- \\
132.43)\end{array}$ & 14 & $\begin{array}{l}10 \\
(71.4)\end{array}$ & 0.05 & $\begin{array}{l}8.94(0.96- \\
82.96)\end{array}$ \\
\hline Housing type & & & 0.35 & & & & 0.17 & \\
\hline $\begin{array}{l}\mathrm{HDB}^{1} 1 \& 2 \text { room(s) } \\
\text { flat }\end{array}$ & 7 & $4(57.1)$ & - & 1.00 & 4 & $1(25.0)$ & - & 1.00 \\
\hline HDB 3 room flat & 19 & $\begin{array}{l}14 \\
(73.7)\end{array}$ & 0.28 & $\begin{array}{l}3.37(0.38- \\
29.97)\end{array}$ & 14 & $\begin{array}{l}11 \\
(78.6)\end{array}$ & 0.02 & $\begin{array}{l}55.72 \\
(1.82- \\
1706.42)\end{array}$ \\
\hline HDB 4 room flat & 36 & $\begin{array}{l}30 \\
(83.3)\end{array}$ & 0.10 & $\begin{array}{l}6.20(0.73- \\
52.80)\end{array}$ & 30 & $\begin{array}{l}17 \\
(56.7)\end{array}$ & 0.12 & $\begin{array}{l}12.38 \\
(0.53- \\
287.48)\end{array}$ \\
\hline HDB 5 room flat & 32 & $\begin{array}{l}26 \\
(81.3)\end{array}$ & 0.11 & $\begin{array}{l}5.68(0.67- \\
47.78)\end{array}$ & 26 & $\begin{array}{l}14 \\
(53.9)\end{array}$ & 0.13 & $\begin{array}{l}10.78 \\
(0.51- \\
227.66)\end{array}$ \\
\hline $\begin{array}{l}\text { Condominium \& } \\
\text { Landed Property }\end{array}$ & 6 & $4(66.7)$ & 0.99 & $\begin{array}{l}0.99(0.06- \\
16.43)\end{array}$ & 4 & $3(75.0)$ & 0.09 & $\begin{array}{l}32.53 \\
(0.57- \\
1848.67)\end{array}$ \\
\hline Duration of DM & & & 0.11 & & & & 0.02 & \\
\hline $0-5$ years & 34 & $\begin{array}{l}23 \\
(67.7)\end{array}$ & - & 1.00 & 23 & $\begin{array}{l}13 \\
(56.5)\end{array}$ & - & 1.00 \\
\hline
\end{tabular}




\begin{tabular}{|c|c|c|c|c|c|c|c|c|}
\hline \multicolumn{2}{|l|}{ Variable } & \multicolumn{7}{|c|}{ Attitude } \\
\hline & & \multicolumn{3}{|c|}{$\begin{array}{l}\text { Participants who agreed } \\
\text { diabetic patients should go } \\
\text { for eye examinations }\end{array}$} & & \multicolumn{3}{|c|}{$\begin{array}{l}\text { Participants who agreed } \\
\text { diabetic patients should } \\
\text { go for annual eye } \\
\text { examinations }\end{array}$} \\
\hline & Total & n (\%) & p-value & $\begin{array}{l}\text { Adjusted } \\
\mathrm{OR}^{2} \\
\left(95 \% \mathrm{Cl}^{3}\right)\end{array}$ & Total & n (\%) & p-value & $\begin{array}{l}\text { Adjusted } \\
\text { OR }(95 \% \\
\text { CI) }\end{array}$ \\
\hline $6-10$ years & 21 & $\begin{array}{l}16 \\
(76.2)\end{array}$ & 0.39 & $\begin{array}{l}1.91 \\
(0.44-8.34)\end{array}$ & 16 & $6(37.5)$ & 0.01 & $\begin{array}{l}0.08 \\
(0.01-0.56)\end{array}$ \\
\hline$>10$ years & 45 & $\begin{array}{l}39 \\
(86.7)\end{array}$ & 0.04 & $\begin{array}{l}4.03(1.09- \\
14.87)\end{array}$ & 39 & $\begin{array}{l}27 \\
(69.2)\end{array}$ & 0.68 & $\begin{array}{l}1.33 \\
(0.35-5.04)\end{array}$ \\
\hline
\end{tabular}

${ }^{1}$ HDB: Housing Development Board

2OR: odds ratio

$395 \% \mathrm{Cl}: 95 \%$ confidence interval

${ }^{4}$ Lower primary: $<6$ years of formal education.

Primary: 6 years of formal education with attainment of Primary School Leaving Examination (PSLE) qualification.

Secondary: $10-11$ years of formal education with attainment of Ordinary or Normal level qualification.

Post-secondary: Attainment of Advanced level and/or higher qualification.

Adjusted for gender, age, ethnic group, education, type of housing and duration of DM.

\section{c. Factors influencing practice patterns (Table 3)}

The practice patterns of participants with regards to visiting an eye doctor despite having good glucose control was significantly higher in those who had more than ten years duration of DM compared to zero to five years duration (OR: $4.05,95 \% \mathrm{Cl}$ : $1.32-12.40$, overall $p$-value: 0.01 , specific $p$-value: 0.01 ). The practice of choosing an eye doctor if participants are diagnosed with DR was significantly higher in those with post-secondary education compared to those with no formal or lower primary education (OR: 12.83; 95\% Cl: 1.83-89.83; overall p-value: 0.03 ). 
Table 3. Associations of practice with participant characteristics, KAP of DM and DR in a tertiary eye clinic in Singapore.

\begin{tabular}{|c|c|c|c|c|c|c|c|}
\hline \multicolumn{2}{|l|}{ Variable } & \multicolumn{6}{|c|}{ Practice } \\
\hline & & \multicolumn{3}{|c|}{$\begin{array}{l}\text { Participants who agreed } \\
\text { they should/will you visit } \\
\text { an ophthalmologist despite } \\
\text { having good blood glucose } \\
\text { control. }\end{array}$} & \multicolumn{3}{|c|}{$\begin{array}{l}\text { Participants who would choose } \\
\text { the ophthalmologist as their } \\
\text { choice of healthcare profession } \\
\text { if they were diagnosed with } \\
\text { diabetic retinopathy. }\end{array}$} \\
\hline & Total & n (\%) & p-value & $\begin{array}{l}\text { Adjusted OR } \\
\left(95 \% \mathrm{Cl}^{3}\right)\end{array}$ & n (\%) & p-value & $\begin{array}{l}\text { Adjusted OR } \\
(95 \% \mathrm{CI})\end{array}$ \\
\hline \multicolumn{8}{|l|}{ Gender } \\
\hline Male & 56 & $\begin{array}{l}40 \\
(71.4)\end{array}$ & - & 1.00 & $\begin{array}{l}38 \\
(67.9)\end{array}$ & - & 1.00 \\
\hline Female & 44 & $\begin{array}{l}26 \\
(59.1)\end{array}$ & 0.73 & $1.22(0.39-3.82)$ & $\begin{array}{l}21 \\
(47.7)\end{array}$ & 0.33 & $0.59(0.21-1.70)$ \\
\hline \multicolumn{8}{|l|}{ Age group (yrs) } \\
\hline $21-44$ yrs & 41 & $\begin{array}{l}33 \\
(80.5)\end{array}$ & - & 1.00 & $\begin{array}{l}31 \\
(75.6)\end{array}$ & - & 1.00 \\
\hline$>60 \mathrm{yrs}$ & 59 & $\begin{array}{l}33 \\
(55.9)\end{array}$ & 0.05 & $0.34(0.12-1.00)$ & $\begin{array}{l}28 \\
(47.5)\end{array}$ & 0.10 & $0.43(0.16-1.17)$ \\
\hline Ethnic group & & & 0.48 & & & 0.40 & \\
\hline Chinese & 71 & $\begin{array}{l}44 \\
(62.0)\end{array}$ & - & 1.00 & $\begin{array}{l}42 \\
(59.2)\end{array}$ & - & 1.00 \\
\hline Malay & 11 & $8(72.7)$ & 0.60 & $1.60(0.27-9.36)$ & $8(72.7)$ & 0.77 & $1.28(0.23-7.09)$ \\
\hline Indian & 18 & $\begin{array}{l}14 \\
(77.8)\end{array}$ & 0.24 & $2.31(0.57-9.37)$ & $9(50.0)$ & 0.20 & $0.44(0.13-1.56)$ \\
\hline $\begin{array}{l}\text { Educational } \\
\text { level }^{4}\end{array}$ & & & 0.22 & & & 0.03 & \\
\hline $\begin{array}{l}\text { No formal } \\
\text { qualifications/ } \\
\text { Lower primary }\end{array}$ & 30 & $\begin{array}{l}13 \\
(43.3)\end{array}$ & - & 1.00 & $\begin{array}{l}10 \\
(33.3)\end{array}$ & - & 1.00 \\
\hline Primary (PSLE) & 37 & $\begin{array}{l}26 \\
(70.3)\end{array}$ & 0.12 & $\begin{array}{l}2.78 \\
(0.77-10.08)\end{array}$ & $\begin{array}{l}23 \\
(62.2)\end{array}$ & 0.03 & $\begin{array}{l}4.23 \\
(1.19-15.04)\end{array}$ \\
\hline $\begin{array}{l}\text { Secondary ('O'//'N' } \\
\text { level) }\end{array}$ & 18 & $\begin{array}{l}14 \\
(77.8)\end{array}$ & 0.13 & $\begin{array}{l}3.45 \\
(0.71-16.84)\end{array}$ & $\begin{array}{l}13 \\
(72.2)\end{array}$ & 0.03 & $\begin{array}{l}5.47 \\
(1.17-25.59)\end{array}$ \\
\hline Post-Secondary & 15 & $\begin{array}{l}13 \\
(86.7)\end{array}$ & 0.08 & $\begin{array}{l}6.30 \\
(0.81-49.01)\end{array}$ & $\begin{array}{l}13 \\
(86.7)\end{array}$ & 0.01 & $\begin{array}{l}12.83 \\
(1.83-89.83)\end{array}$ \\
\hline Housing type & & & 0.96 & & & 0.40 & \\
\hline
\end{tabular}




\begin{tabular}{|c|c|c|c|c|c|c|c|}
\hline \multicolumn{2}{|l|}{ Variable } & \multicolumn{6}{|c|}{ Practice } \\
\hline & & \multicolumn{3}{|c|}{$\begin{array}{l}\text { Participants who agreed } \\
\text { they should/will you visit } \\
\text { an ophthalmologist despite } \\
\text { having good blood glucose } \\
\text { control. }\end{array}$} & \multicolumn{3}{|c|}{$\begin{array}{l}\text { Participants who would choose } \\
\text { the ophthalmologist as their } \\
\text { choice of healthcare profession } \\
\text { if they were diagnosed with } \\
\text { diabetic retinopathy. }\end{array}$} \\
\hline & Total & n (\%) & p-value & $\begin{array}{l}\text { Adjusted OR } \\
\left(95 \% \mathrm{Cl}^{3}\right)\end{array}$ & n (\%) & p-value & $\begin{array}{l}\text { Adjusted OR } \\
(95 \% \mathrm{Cl})\end{array}$ \\
\hline $\begin{array}{l}\mathrm{HDB}^{1} 1 \& 2 \\
\text { room(s) flat }\end{array}$ & 7 & $5(71.4)$ & - & 1.00 & $4(57.1)$ & - & 1.00 \\
\hline HDB 3 room flat & 19 & $\begin{array}{l}11 \\
(57.9)\end{array}$ & 0.74 & $0.67(0.07-6.87)$ & $\begin{array}{l}13 \\
(68.4)\end{array}$ & 0.18 & $\begin{array}{l}4.40 \\
(0.52-37.28)\end{array}$ \\
\hline HDB 4 room flat & 36 & $\begin{array}{l}23 \\
(63.9)\end{array}$ & 0.84 & $0.79(0.08-7.71)$ & $\begin{array}{l}21 \\
(58.3)\end{array}$ & 0.43 & $\begin{array}{l}2.29 \\
(0.30-17.51)\end{array}$ \\
\hline HDB 5 room flat & 32 & $\begin{array}{l}22 \\
(68.8)\end{array}$ & 0.98 & $0.97(0.10-9.72)$ & $\begin{array}{l}18 \\
(56.3)\end{array}$ & 0.67 & $\begin{array}{l}1.55 \\
(0.21-11.72)\end{array}$ \\
\hline $\begin{array}{l}\text { Condominium } \\
\text { and Landed } \\
\text { Property }\end{array}$ & 6 & $5(83.3)$ & 0.77 & $\begin{array}{l}1.60 \\
(0.07-36.54)\end{array}$ & $3(50.0)$ & 0.73 & $0.64(0.05-8.55)$ \\
\hline Duration of DM & & & 0.01 & & & 0.65 & \\
\hline $0-5$ years & 34 & $\begin{array}{l}16 \\
(47.1)\end{array}$ & - & 1.00 & $\begin{array}{l}19 \\
(55.9)\end{array}$ & - & 1.00 \\
\hline 6-10 years & 21 & $\begin{array}{l}18 \\
(85.7)\end{array}$ & 0.006 & $\begin{array}{l}10.62 \\
(1.99-56.59)\end{array}$ & $\begin{array}{l}15 \\
(71.4)\end{array}$ & 0.43 & $1.79(0.43-7.42)$ \\
\hline$>10$ years & 45 & $\begin{array}{l}32 \\
(71.1)\end{array}$ & 0.01 & $\begin{array}{l}4.05 \\
(1.32-12.40)\end{array}$ & $\begin{array}{l}25 \\
(55.6)\end{array}$ & 0.92 & $0.95(0.32-2.78)$ \\
\hline
\end{tabular}

HDB: Housing Development Board

${ }^{2}$ OR: odds ratio

${ }^{3}$ 95\% Cl: 95\% confidence interval

${ }^{4}$ Lower primary: $<6$ years of formal education.

Primary: 6 years of formal education with attainment of Primary School Leaving Examination (PSLE) qualification.

Secondary: 10 - 11 years of formal education with attainment of Ordinary or Normal level qualification.

Post-secondary: Attainment of Advanced level and/or higher qualification.

Adjusted for gender, age, ethnic group, education, type of housing and duration of DM.

Impact of education on KAPP (Table 4):

Knowledge 
Significant improvement was seen in certain domains under knowledge after education as illustrated in Table 1 and summarized below:

1. Knowledge of DM: increased from $57.0 \%$ to $74.0 \%$ ( $p$-value: $<0.001)$;

2. Knowledge of DR: increased from $29.0 \%$ to $84.0 \%$ ( $p$-value: $<0.001$ );

3. Participants who agreed that controlled DM can still lead to diabetic-related eye problems: increased from $35.0 \%$ to $50.0 \%$ ( $p$-value: 0.02 );

4. Participants who correctly knew that they had or did not have DR: increased from $15.0 \%$ to $33.0 \%$ (p-value: $<0.001)$.

After educating patients about DR, more participants attributed DM as causing other eye problems in addition to DR (Table 1). They were also more aware of the severity of their DR. However, these findings were not statistically significant.

\section{Attitude}

Significant improvements in their attitude were seen in:

1. Participants who agreed diabetic patients should go for eye examinations: increased from $78.0 \%$ to $93.0 \%$ ( $p$-value: $<0.001$ );

2. On an annual basis usually: increased from $46.0 \%$ to $55.0 \%$ (p-value 0.02 ).

\section{Practice Patterns}

Similarly, significant improvements in their practice patterns were seen in:

1. Participants who agree that they should/will visit an eye doctor even if they had good blood glucose control: increased from $66.0 \%$ to $80.0 \%$ (p-value: 0.01 );

2. Participants who would choose an eye doctor as the healthcare professional in the event they are diagnosed with DR: increased from $59.0 \%$ to $79.0 \%$ ( $p$-value: $<0.001$ ).

Collectively, participants with prior knowledge of DR demonstrated greater impact from education contributing to better attitude and practice patterns - showing an increment of more than $10 \%$ - in realizing the importance of having an eye examination and preferring an eye doctor for the eye examination.

Table 4. Comparison between pre- and post-KAP levels, KAP of DM and DR in a tertiary eye clinic in Singapore.

\begin{tabular}{|l|l|l|l|}
\hline Variable & Pre-KAP & Post-KAP & p-value \\
\hline \multicolumn{2}{|l|}{ n (\%) } & n (\%) & \\
\hline Knowledge & $57(57.0)$ & $74(74.0)$ & $<0.001$ \\
\hline Knowledge of DM $(n=100)$ & $29(29.0)$ & $84(84.0)$ & $<0.001$ \\
\hline Knowledge of DR $(n=100)$ & $35(35.0)$ & $50(50.0)$ & 0.02 \\
\hline $\begin{array}{l}\text { Participants who agreed that individuals } \\
\text { with controlled DM can still have } \\
\text { diabetic-related eye problems. ( }=100)\end{array}$ & & & \\
\hline $\begin{array}{l}\text { Participants who agreed that apart from } \\
\text { retina being affected in DM, DM can still } \\
\text { cause: }(\mathrm{n}=100)\end{array}$ & & $7(7.0)$ & 0.06 \\
\hline Eye movements/ muscles problems & $2(2.0)$ & & \\
\hline
\end{tabular}




\begin{tabular}{|c|c|c|c|}
\hline Variable & Pre-KAP & Post-KAP & p-value \\
\hline & n (\%) & n (\%) & \\
\hline Cataract & $23(23.0)$ & $12(12.0)$ & 0.03 \\
\hline Glaucoma & $7(7.0)$ & $20(20.0)$ & 0.01 \\
\hline All of the above & $27(27.0)$ & $34(34.0)$ & 0.09 \\
\hline Don't know & $40(40.0)$ & $25(25.0)$ & 0.01 \\
\hline $\begin{array}{l}\text { Participants who think they know they } \\
\text { have/ do not have Diabetic Retinopathy. } \\
(\mathrm{n}=88)\end{array}$ & $18(20.5)$ & $46(52.3)$ & $<0.001$ \\
\hline $\begin{array}{l}\text { Number of subjects correctly knowing } \\
\text { they have/do not have DR. }(n=88)\end{array}$ & $15(17.1)$ & $33(37.5)$ & $<0.001$ \\
\hline $\begin{array}{l}\text { Number of participants who think they } \\
\text { know they have DR, correctly knowing } \\
\text { the grade of Diabetic Retinopathy they } \\
\text { have. }\end{array}$ & & & \\
\hline $\mathrm{n}$ & 12 & 35 & \\
\hline Correct match & $4(33.3)$ & $9(25.7)$ & 0.06 \\
\hline \multicolumn{4}{|l|}{ Attitude } \\
\hline $\begin{array}{l}\text { Participants who agreed that diabetes } \\
\text { patients should go for eye examinations. } \\
(\mathrm{n}=100)\end{array}$ & $78(78.0)$ & $93(93.0)$ & $<0.001$ \\
\hline \multicolumn{4}{|l|}{$\begin{array}{l}\text { Participants who agreed that diabetic } \\
\text { patients should go for eye examinations: } \\
(\mathrm{n}=78)\end{array}$} \\
\hline Once a year & $46(59.0)$ & $55(70.5)$ & 0.02 \\
\hline More than once a year & $32(41.0)$ & $23(29.5)$ & \\
\hline \multicolumn{4}{|l|}{ Practice } \\
\hline $\begin{array}{l}\text { Participants who agreed they should/ } \\
\text { will you visit an ophthalmologist even } \\
\text { if they had good blood glucose control. } \\
(n=100)\end{array}$ & $66(66.0)$ & $80(80.0)$ & 0.01 \\
\hline $\begin{array}{l}\text { Participants who would choose } \\
\text { ophthalmologist as their choice of } \\
\text { healthcare profession if they were } \\
\text { diagnosed with diabetic retinopathy ( } \\
=100 \text { ) }\end{array}$ & $59(59.0)$ & $79(79.0)$ & $<0.001$ \\
\hline
\end{tabular}




\section{Discussion}

Our results indicate that the level of knowledge about DM and DR among the participants were variable. More than half of the respondents had prior knowledge of DM, but only less than one-third of them were aware of DR. This is comparable to other studies that reported poor knowledge of DR among diabetic patients. Reduced knowledge about DR could translate into greater risk of potentially sightthreatening complications from DR owing to ignorance and indifference. This is supported by the fact that participants with better knowledge of DM and DR are more likely to have correct health-seeking behavior such as better control of glycosylated haemoglobin ( $\mathrm{HbA} 1 \mathrm{c}$ ) levels ${ }^{8}$ and regular eye assessments by ophthalmic professionals. ${ }^{9}$ The latter being especially crucial since it can reduce blindness up to $98 \%$ via early detection of DR. ${ }^{9}$ Unfortunately, many studies have reported low utilization of ophthalmic services in diabetic patients. ${ }^{10}$ Could this be attributed to poor knowledge or awareness, which can pose a threatening public health issues in view of the anticipated rise in the prevalence of DM and DR in Asia. ${ }^{11}$

In our study, the participant characteristics that led to better knowledge of DR, attitudes and practice patterns were higher educational level and longer duration of $D M$. A local study ${ }^{5}$ similarly showed that a longer duration of DM was associated with better knowledge of DR. Such association could be accounted for by increased exposure to doctor-patient interaction/education and thus increased adherence to regular eye examinations. ${ }^{1}$. It calls for a need to inculcate knowledge, correct attitudes and practice patterns at the beginning and/or early into the diagnosis of $D M$. This is especially pertinent when the prevalence of $D R$ is high even in newly diagnosed diabetic patients. ${ }^{13}$

We also found a significantly higher proportion of Singaporean Indians to have knowledge of DM compared to Singaporean Chinese. This could be due to increased awareness of DM among Indians due to its high prevalence among this ethnic group. ${ }^{14}$

Contrary to our expectations, we did not find any association between higher educational level and higher socio-economic status (represented by housing type) with knowledge of DR as seen in previous Asian studies. ${ }^{16-18}$ This may be an important finding since it suggests that DR education is indeed lacking in the overall community. Interestingly, a high percentage of DR was noted in participants with post-secondary education and living in condominium/landed properties at $53.3 \%$ and $50.0 \%$ respectively.

Many KAPP studies specific ${ }^{15,19}$ to DM and DR have found education to positively impact KAPP. Tham et al.' found diabetic patients who had attended a diabetic education program or counselling session showed a $9.4 \%$ increase in knowledge of DM and its complications. In our study, significant improvement was seen in most of the knowledge section and all of the attitude and practice patterns sections after twenty minutes of education using the booklet. This shows that:

1. Even a short duration of education is beneficial;

2. The current educational DM and DR booklet is useful;

3. Pamphlets/booklets are useful educational tools by themselves, even 
without formal educational programs or counseling sessions. The strengths of our study include the following:

1. Being a pilot KAPP study on DM and DR across all ethnic groups in Singapore;

2. The assessment of impact of education on KAPP is another pilot initiative and highlight;

3. An excellent response rate of $92.6 \%$ in our data consolidation.

Alhough our study sample of diabetic patients is a good representation of the general DM population in Singapore, ${ }^{14}$ the sample population could have been more, which we think is the limitation of this pilot study.

To conclude, in light of the above findings, the following recommendations can be made:

1. A fifteen to thirty minutes education using existing DM and DR educational booklet to all newly-diagnosed diabetics.

2. This can be followed-up by formal educational programs or counseling sessions.

3. Use of mass media to create awareness of $D M$ and $D R$, since there is a general lack of knowledge of DM and DR among the diabetic population and possibly the general community.

4. Considerations to reduce cost of ophthalmology outpatient fees, especially for follow-up of chronic eye conditions such as DR, to encourage increased adherence to regular eye examination.

These measures may contribute to reducing DR complications and the attendant plight and menace of DR related blindness.

\section{Acknowledgement}

The Singapore Health Promotion Board kindly agreed with the use of the booklet, Management of Diabetic Retinopathy.

\section{References}

1. Tham KY, Ong JJ, Tan DK, How KY. How much do diabetic patients know about diabetes mellitus and its complications? Ann Acad Med Singapore 2004;33:503-509.

2. Funatsu $\mathrm{H}, \mathrm{Hori} \mathrm{S}$, Shimuzu E, Nakamura S. Questionnaire survey on periodic ocular examination in Japanese diabetic patients. Am J Ophthalmol 2003;136:95-97.

3. Khandekar R, Shah S, Al Lawatti J. Retinal examination of diabetic patients: knowledge, attitudes and practices of physicians in Oman. Revue de Santé de la Méditerranée orientale 2008;14:850-857.

4. James SM, Henry SN, Philip R, Emmae R, Mya A, San M, et al. Awareness of diabetic eye disease among general practitioners and diabetic patients in Yangon, Myanmar. Clin Experiment Ophthalmol 2008;36:265-273.

5. Huang OS, Tay WT, Tai ES, Wang J.J, Saw S.M, Jeganathan V.S.E et al. Lack of Awareness amongst Community Patients with Diabetes and Diabetic Retinopathy: The Singapore Malay Eye Study. Ann Acad Med Singapore 2009;38:1048-1055.

6. Management of Diabetic Retinopathy [Internet]. Health Promotion Board, Singapore 2008. [cited 201325 Mar]. Available from: http://www.hpb.gov.sg/edumaterials/default.aspx?pgno=18

7. Early Treatment of Diabetic Retinopathy Study Research Group: Grading diabetic retinopathy from stereoscopic colour fundus photographs - an extension of the modified Airlie House Classification. ETDRS Report 10. Ophthalmology 1991;98:786-806. 
8. Naresh B, Kim, Bharat R, Sachin T. Diabetes and Diabetic Retinopathy: Knowledge, Attitude, Practice (KAP) Among Paramedical Personnel (PMPS) and Community Members (CMS) In Southern India. AIOC Proceedings 2009;150-152.

9. Rohan TE, Frost CD, Wald NJ. Prevention of blindness by screening for diabetic retinopathy: a quantitative assessment. BMJ (Clin Res Ed) 1989;299:1198-1201.

10. Muller A, Vu HT, Ferraro JG, Keeffe JE, Taylor HR. Utilization of eye care services in Victoria. Clin Experiment Ophthalmol 2006;34:445-448.

11. Dandona L, Dandona R, Naduvilath TJ, McCarty CA, Rao GN. Population based assessment of diabetic retinopathy in an urban population in southern India. Br J Ophthalmol 1999;83:937-940.

12. Schoenfeld ER, Greene JM, Wu SY, Leske MC. Patterns of adherence to diabetes vision care guidelines: baseline findings from the Diabetic Retinopathy Awareness Program. Ophthalmology 2001;108:563-571.

13. Wong TY, Cheung N, Tay WT, Wang JJ, Aung T, Saw SM, et al. Prevalence and risk factors for diabetic retinopathy: the Singapore Malay Eye Study. Ophthalmology 2008;115:1869-1875.

14. National Health Survey (NHS) 2010 [Internet]. Ministry of Health, Singapore. c2012 - [cited 2013 Mar 25]. Available from: http://www.moh.gov.sg/content/dam/moh_web/Publications/ Reports/2011/NHS2010\%20-\%20low\%20res.pdf

15. Nazl A, Kenan K, Tanju G. The Impact of Patient Education on Diabetes Empowerment Scale (DES) and Diabetes Attitude Scale (DAS-3) in Patients with Type 2 Diabetes. Turk J Med Sci 2008;38:49-57.

16. Rani PK, Raman R, Subramani S, Perumal G, Kumaramanickavel G, Sharma T. Knowledge of diabetes and diabetic retinopathy among rural populations in India, and the influence of knowledge of diabetic retinopathy on attitude and practice. Rural and Remote Health 2008;8:838.

17. Williams MV, Baker DW, Parker RM, Nurss JR. Relationship of functional health literacy to patients' knowledge of their chronic disease. A study of patients with hypertension and diabetes. Archives of Internal Medicine 1998;158:166-172.

18. Rothman RL, Malone R, Bryant B. The spoken knowledge in low literacy in diabetes scale. The Diabetes Educator 2005;31:215-224.

19. Ranabir Pal, Shrayan Pal, Ankur Barua, MK Ghosh. Health education intervention on diabetes in Sikkim. Indian J of Endocrinology and Metabolism 2011;14:3-7. 